\title{
Extended Use of Smartphone Technology Cause Repetitive Upper Limb and Related Stress-strain Injuries and it's Impact on Home-isolation Covid-19 (Pandemic) Affected Population: Short Communication
}

\author{
Manashi Dey* \\ Institute of Physiotherapy, Vydehi Institute of Physiotherapy, RGUHS, Bangalore, \\ India \\ *Corresponding Author: Manashi Dey, Institute of Physiotherapy, Vydehi Institute \\ of Physiotherapy, RGUHS, Bangalore, India.
}

DOI: 10.31080/ASOR.2022.05.0432
Received: February 14, 2022

Published: February 28, 2022

(C) All rights are reserved by Manashi Dey.

\section{Abstract}

COVID-19 pandemic has resulted in a devastating threat to humans primarily in terms of lifestyle, economy and health and, majorly affecting the mental health, posture, associated pains mainly upper body pain, low back pain or joint pains and accompanied with lethargy and high amount of exhaustion. Population, who were mild-moderate affected were asked to be bed rest with prophylactic treatment and on home-isolation, if affected severely, were admitted to hospital setting for antibiotic therapy. Set of people who were into home-isolation, however, due western society lifestyle behaviors like physical inactivity and sedentariness made them more inclined towards cellular technology. Due to this pandemic or otherwise this "smart-cellular technology" has made a strong hold in ourselves. The usage of smartphone technology has increased drastically during the national lockdown to keep themselves updated in terms of health information, education, socialization, banking solution, household remedies. With this usage of smartphone technologies came up "Text Claw", Blackberry Thumb or WhatsAppitis or "prolonged-phone-posture" leading to neck, elbow, wrist or finger referred or primary pain areas, further damaging any particular nerve or leading to syndrome like conditions. The usage of smartphone technology is greatly aggravated by the containment measures imposed to control the expansion of the pandemic.

Keywords: Musculoskeletal Disorders; COVID-19; Health; Muscle; Pain; Injury; Smartphone Technology; Home-isolation

\section{Abbreviations}

Smartphone Technology, Coronavirus Disease 2019, Telemedicine, Fifth Generation, Repetitive Strain Injury

\section{Introduction}

Coronavirus disease 2019

The coronavirus disease 2019 (COVID-19) pandemic spread all across India and globally, occurred at a time of global digitalization that was characterized by quick connection among people and information required from anywhere in the world [3]. This led affected and unaffected population during the national lockdown, to spend more time at home or at community-based centres during recovery phase and with major use of technological devices; use of SMT increased [10]. Most people, who fell sick during this COVID-19 lockdown phase, experienced mild to moderate symptoms and they recovered without any special treatment and thus recovered at home setting, (stay-at-home or self-isolate until they recovered). However, some, became seriously-ill and required medical attention at hospital setting. Those who underwent self-isolation became more inclined towards usage of SMT.

At present, the use of smartphone technology has increased as people look for quick-accurate update about COVID-19 pandemic or for work opportunities. Some articles, show the challenges faced during the COVID-19 pandemic and assessed the extended role of 
Smartphone technology (SMT) in support to the use of Telemedicine (TM) in various applications at various levels.

\section{Smartphone technology (SMT)}

A smartphone, is a newer class of cellular telephone with an integrated-computer-technology and other features such as an operating system, web browsing, and ability to run various software applications. They are referred as 'Smart' as they can provide information as and when needed at the touch of our fingers and can be used across widely in most convenient way, by various age categories. Currently, smartphones are equipped with various technical features (camera, video recording, Global Positioning Service (GPS) navigation, and games, sending and receiving Electronic mail (email), web search applications for various purposes). SMT has influenced areas such as health, social life, business, education, banking and further more [2].

COVID-19 generated a seismic shift in our use of wireless technology. Educating, homeworking, communicating, socializing and entertainment have all become online pursuits during the COVID-19 lockdown phase. This is trending even after lockdown phase, seen all across the globe. Physically distanced have turned to more usage of online technologies for work, health education, and socialization with friends and family and entertainment [21]. Every generation have been seen going through this. One study showed that $71 \%$ of school children used more of SMT. while a UK survey reported online video calls made by the over 65 s went up $22 \%$ in February and 61\% in May [20].

To this, increased demand to new spectrums, 5G smartphone devices are a step forward in improving both patient care and physician competence, as well as decreasing resource consumption, which possibly may reduce healthcare costs. Advances in technology empowered the ability to respond to the COVID-19 pandemic.

5G SMT phone with Artificial intelligence features can contribute to the fight against COVID-19 by i) early warnings and alerts, ii) tracking and prediction, iii) data dashboards, iv) diagnosis of COVID-19 patients determine best treatment plan and predict postoperative complications to enable early interventions when necessary, v) prognosis, treatments, and cures, and vi) social control [16].
Primary or secondary pain in neck, shoulders, wrists and fingers (ring and pinky fingers) are common and more frequently reported upper limb musculoskeletal areas due to overuse of the smartphone [4], for the COVID-19 affected population.

Utilizing of Smartphone technology (SMT) during COVID-19 phase

Smart phone technology is the extended application of TM with an advantage of portability, with several features at tips of the fingers [12] answer a number of important epidemiological questions needed to guide the implementation and evaluation of various interventions. SMT applications and COVID-19 pandemic play various role in health-care setup, trauma and orthopaedics surgery applications, radiodiagnosis, mental-health, training in education, group-counselling, triage, teleconsultation remotely, monitoring of COVID-19, Aarogya Setu COVID-19 tracking app.

A key advantage of SMT is the possibility to quickly collect bespoke data in many areas; particularly in a pandemic, where tailored responses to specific epidemiologic and social contexts were required. Use of mobile phone data might require fewer assumptions about the transportability of derived mobility and contact metrics across different populations.

The use of cell phone technology or SMT to deliver health care services to COVID-19 affected population uses mHealth (Mobile health). The advent of SMT has increased the potential impact of mHealth intensely and also has positive implications for COVID-19 patients' health [1].

SMT are also used to undertake VFC and also to monitor the chronic orthopaedic conditions such as osteoarthritis during COVID-19 pandemic period, when specially elderly affected group were unable to travel, due to Lockdown restrictions $[7,10]$. Virtual Fracture Clinics (VFC) were another-newer way of assessing and managing the reported musculoskeletal injuries if occurred COVID-19 [9].

With high speed SMT, remotely controlled surgery (Telesurgery) will be a step closer due to much improved image quality and real-time video communication [14]. 
Information and communication technologies helps us in diagnosis, prevention and advice for the management of acute musculoskeletal injuries or chronic orthopaedic conditions [9].

\begin{tabular}{|l|c|c|}
\hline S. No & Devices/Platforms & Examples \\
\hline a. & Video platforms & Zoom, Skype, Facetime (iPhone) \\
\hline b. & Audio platforms & telephone calls, advice etc. \\
\hline c. & Text platforms & Messaging, chat mode \\
\hline d. & Wearable devices & $\begin{array}{c}\text { such as the Apple Watch, WHOOP } \\
\text { Stratbit, Zephyr BioHarness, } \\
\text { or VivaLNK Vital Scout }\end{array}$ \\
\hline
\end{tabular}

Table 1

Studies have shown that smartphone use have helped in avoiding unnecessary visits to the tertiary hospital-setup and also decreased the incidence of missed diagnosis of fractures in patients in general practice [19].

As more and more population of different categories become accustomed with working remotely and also children are getting habituated to home - schooling, there has been increased demand for smart home devices.

\section{Repetitive strain injury}

RSI is a term referred to discrete conditions associated with repetitive tasks, and sustained awkward positions. Cause is related to overuse of the muscles and tendons in the upper body [6].

\section{Communication modes with SMT}

The use of wearable sensors toward monitoring activity levels could provide an objective means of staying physically active and healthy during the COVID-19 pandemic. There is a growing amount of evidence showing a clear downward trend in recovery scores in the days leading up to the onset of COVID-19 symptoms [5].

Side effects of SMT (Commonly associated conditions of upper body with repeated overuse of smart phones/SMT as smart home devices)

- The COVID-19 pandemic has therefore led to an increased push to use of SMT leading to pain and weakness of certain structures.

- $\quad$ Putting neck and upper limb in uncomfortable positions for a prolonged period of time, leading to strains and overuse injuries. Causing pain in neck (cervical region), upper back (thoracic region), shoulder (upper and lower fibres of trapezius), and upper limb (shoulder, elbow, hands and thumb) and numbness and tingling in hands and fingers.

- At cervical spine, shoulder and thoracic region, due to bad posture while lying or sitting with neck forward bending leading to neck pain (Text Neck), shoulder pain and Fibromyalgia.

- Associated condition at elbow region is Cubital Tunnel syndrome or "Text Claw". Also called "Cell Phone Elbow", Textclaw is a non-medical term that describes cramping of all of the fingers and aching of the muscles that come from constant use of SMT while gaming, scrolling or/and texting. Described with numbness or tingling in the ring and pinky fingers that occurs after when the elbow is bent for long period [6]. Cell phone elbow/prolonged-phone-posture (PPP) occur due to bending or flexed posture of the elbow for long period of time, while using the phone for audio call. These positions may progressively irritate the ulnar nerve [18].

- With overuse of muscles and tendons at wrist and hand region leads to texting tenosynovitis. Triggered by a Stenosing inflammation of the tendon sheath of the muscles (abductor pollicis longus and extensor pollicis brevis) in the first dorsal compartment. Redundant movements of the thumb for texting, messaging or gaming require repetitive that irritates the tendon sheath. Pain over the surface of the radial aspect of the wrist and weakness and dysesthesias (numbness, tingling, burning, and cramping) over the base of the thumb/wrist [17]. Other encountered problems of muscles and tendons of this area - De-quervain's Tenosynovitis (Texting tenosynovitis, or Blackberry Thumb or WhatsAppitis), Extensor tendinitis, carpal tunnel syndrome, extensor digitorum communis, extensor pollicis longus tendinitis/rupture (swiper's thumb and I-pad hand), myofascial syndrome of adductor pollicis, pain in 1 st interosseous region, weakness of grip or pinch.

- $\quad$ Prior work show neck pain can be sometimes commonly seen associated with low back ache or as referred pain travelling from upper thoracic or cervical region to lumbar area due to constant confining to bed or bad posture while sitting in chair while home-education or working.

Determining the appropriate resolution for using the technology, how these can change (Good to know guidance/recommendations for appropriate use of Smart phone). 
Extended Use of Smartphone Technology Cause Repetitive Upper Limb and Related Stress-strain Injuries and it's Impact on Home-isolation Covid-19 (Pandemic) Affected Population: Short Communication

\begin{tabular}{|c|c|c|}
\hline S. No. & Do's & Do not's \\
\hline a. & support the forearms & $\begin{array}{l}\text { Avoid sitting with the } \\
\text { head bent forward }\end{array}$ \\
\hline b. & use of both the thumbs & $\begin{array}{c}\text { Avoid texting with high } \\
\text { speed }\end{array}$ \\
\hline c. & $\begin{array}{c}\text { use of smartphone while } \\
\text { sitting (and not while } \\
\text { walking(brisk walking/jog)) }\end{array}$ & Avoid elbow bending \\
\hline d. & $\begin{array}{l}\text { keep switching the hands } \\
\text { intermittently, while using } \\
\text { SMT and giving rest to the } \\
\text { hand }\end{array}$ & $\begin{array}{l}\text { Avoid use of smartphone } \\
\text { whilst driving or during } \\
\text { morning/evening walks }\end{array}$ \\
\hline e. & $\begin{array}{l}\text { keeping the wrists straight } \\
\text { while using SMT }\end{array}$ & $\begin{array}{l}\text { Avoid marathon brows- } \\
\text { ing or messaging session } \\
\text { (commonly seen these } \\
\text { days in youngsters) }\end{array}$ \\
\hline c. & $\begin{array}{l}\text { try to hold phone in both the } \\
\text { hands }\end{array}$ & $\begin{array}{l}\text { Avoid usage of smart } \\
\text { phone for more than } \\
\text { 15-20 minutes, at a } \\
\text { stretch }\end{array}$ \\
\hline
\end{tabular}

Table 2

\section{Limitations of SMT}

It is important to know that many mobile applications and SMT devices currently lack the certain parameters which are required to make them reliable and viable tools. Like any other digital health innovation, Mobile health technology is no exception. It also faces privacy and data ownership issues [15]. The challenges it poses could in itself be motivation for further developments and innovations [2].

\section{Results and Discussion}

This study, included a number of patients affected by moderatesevere COVID-19, underwent home-isolation, provided some relevant findings of pain areas in neck, upper thoracic, wrist and pinky finger and $1^{\text {st }}$ interosseous space due to provoking overuse of SMT while lying or sitting. First, we reported a high incidence of pinky and ring finger and thumb pain, despite the recommended prophylaxis. Second, patients under community-based centres/shelter showed a 6 times increased pain area specially of neck region, due to more confinement to bed and bad lying posture compared with those who were at home isolation, were at ease to move around the home or self-isolation area. Third, COVID-19 patients who developed acute and constant elbow or wrist pain during home-isolation reported more usage of SMT in terms of banking solutions, education, online transactions or online purchase showed an increased risk of constant pain or strain or injury compared to those who did not experience such type of pain/strain/injury, while being admitted in hospital setting, as they were not permitted to use SMT as it might cause noise or any other disturbance to the ward/unit patients. This article motivates to investigate further about various smartphone technologies related upper body injuries/strain for the pandemic response. A term 'Plasticity' describes the ability of our muscles to adapt to variations in activity and in working demand. This adaptive event involves the whole muscle fiber structure from the sarcolemma to the mitochondria, including the myofibrils, the extracellular matrix, as well as capillaries surrounding the muscle fibers [13]. With the advancement, the use of smartphone technology opens up for exciting and new opportunities like global-smart healthcare systems, connected world, and smart education systems, further leading to pain-free usage of smartphone technologies. Exercise is one of the most frequently prescribed therapy [8]. Therefore, it is necessary to further investigate digital/smartphone technologies amid COVID-19 that will lead to the future resilient and sustainable smart communities. Adoption of wearable sensors (and digital health technologies overall) in United States towards remote patient monitoring is the issue of data privacy, data sharing, and underreporting. Underreporting of data by some populations may require their consent for safe data sharing and privacy agreements so that it can be used to inform better care, thus decreasing health issues. Moreover, have also discussed the side-effects/hazards posed by SMT, such as privacy, security, and threat of mis-information. Additionally, showed that smartphone technologies are assisting in the survival of the global economy by aiding industries, supply chains, and e-commerce with automation.

\section{Conclusion}

SMT plays a quite significant role in winning the fight against pandemic's disruption of every-day life and looking forward to the "new normal". Smartphone technologies have been extensively used worldwide for the last couple of decades, specially, more during the COVID-19 pandemic. Recently found numerous orthopaedics related stress or strain injuries due to constant or poor way of applications of SMT during COVID-19 pandemic. These phones do help us in avoiding the face-to-face contact (physical distancing) of healthcare provider with the affected population and thus maintain social distancing and avoid virus transmission. Also, 
these SMT are valuable in making the clinical evaluation, diagnosis, timely advice, prescription and also in monitoring of the patients from their home-isolation and in remote areas (community shelter). If SMT are used correctly and with due care, repetitive stress injuries to the musculoskeletal system can be avoided [2]. Someone who gets this often has a predisposition to have the problem [6].

In this study, have discussed about the variety of smartphone technologies challenges stemming due to the COVID-19 pandemic, and have highlighted the need for communication technologies in facing challenges such as monitoring the spread of the virus, healthcare enablement, and allowing virtual education and conferencing. Also, highlighted several use cases that revolutionized SMT during the pandemic, promising a technological transformation in the future. Hence, see an opportunity to design a device that can accurately monitor many or all metrics of pain-interest and through machine learning is able to develop an algorithm to reliably detect changes in population health status.

\section{Conflict of Interest}

The author declares that the study was conducted in the absence of any commercial or financial relationships that could be construed as a potential conflict of interest.

\section{Bibliography}

1. Wilson Kumanan. "Mobile cell phone technology puts the future of health care in our hands". Canadian Medical Association Journal 190.13 (2018): E378-E379.

2. Karthikeyan Iyengar Gaurav K Upadhyaya., et al. "COVID-19 and applications of smartphone technology in the current pandemic". Diabetes and Metabolic Syndrome 14.5 (2020): 733-737.

3. Ma H., et al. "A single-center, retrospective study of COVID-19 features in children: a descriptive investigation". BMC Medicine 18.1 (2020): 123.

4. Kim HJ and Kim JS. "The relationship between smartphone use and subjective musculoskeletal symptoms and university students". The Journal of Physical Therapy Science 27.3 (2015): 575-579.

5. Dhruv R Seshadri., et al. "Wearable Sensors for COVID-19: A Call to Action to Harness Our Digital Infrastructure for Remote Patient Monitoring and Virtual Assessments". Frontiers in Digital Health (2020).
6. Roger Powell MD. "Effects of Smartphones on our Fingers, Hands and Elbows". The Orthopaedic Institute (2020).

7. Iyengar $\mathrm{K}$ and El-Nahas W. "A brief guide to telephone medical consultation". British Journal of Healthcare Management (2020).

8. Bowden Davies KA., et al. "Reduced physical activity in young and older adults: metabolic and musculoskeletal implications". Therapeutic Advances in Endocrinology and Metabolism (2019): 10.

9. Logishetty $\mathrm{K}$ and Subramanyam S. "Adopting and sustaining a Virtual Fracture Clinic model in the District Hospital setting: a quality improvement approach". BMJ Quality Improvement Reports 6.1 (2017).

10. Greenhalgh T., et al. "Video consultations for covid-19". British Medical Journal 368 (2020): m998.

11. Montag C and Elhai JD. "Discussing digital technology overuse in children and adolescents during the COVID-19 pandemic and beyond: on the importance of considering affective neuroscience theory". Addictive Behaviors Reports 12 (2020): 100313.

12. Sarwar M and Soomro TR. "Impact of smartphone's on society". European Journal of Scientific Research 98 (2013): 216226.

13. Pette D. "Historical Perspectives: plasticity of mammalian skeletal muscle". Journal of Applied Physiology 90 (2001): 1119-1124.

14. De Montjoye YA., et al. "OpenPDS: protecting the privacy of metadata through Safe Answers". PLoS One 9.7 (2014).

15. Vaishya R., et al. "Artificial Intelligence (AI) applications for COVID-19 pandemic". Diabetes and Metabolic Syndrome 14.4 (2020): 337-339.

16. Ashurst JV., et al. "Tenosynovitis caused by texting: an emerging disease". The Journal of the American Osteopathic Association 110.5 (2010): 294-296.

17. Darowish M., et al. "What is cell phone elbow, and what should we tell our patients?" Cleveland Clinic Journal of Medicine 76.5 (2009): 306-308.

18. Jacobs JJ., et al. "Fracture diagnostics, unnecessary travel and treatment: a comparative study before and after the introduction of tele radiology in a remote general practice". BMC Family Practice 16 (2015): 53. 
19. Children significantly increase use of technology during lockdown and Enjoy later life by staying healthy and connected from home Wireless Technology and the Impact of COVID-19.

\section{Assets from publication with us}

- Prompt Acknowledgement after receiving the article

- Thorough Double blinded peer review

- Rapid Publication

- Issue of Publication Certificate

- High visibility of your Published work

Website: www.actascientific.com/

Submit Article: www.actascientific.com/submission.php

Email us: editor@actascientific.com

Contact us: +919182824667 